\title{
EXTENSION OF THE MULTIPLE ERDÉLYI-KOBER OPERATOR AND REPRESENTATIONS OF THE GENERALIZED HYPERGEOMETRIC FUNCTIONS
}

\author{
D.B. Karp ${ }^{1}$, J.L. López ${ }^{2}$
}

\begin{abstract}
In this paper we investigate the extension of the multiple Erdélyi-Kober fractional integral operator of Kiryakova to arbitrary complex values of parameters by the way of regularization. The regularization involves derivatives of the function in question and the integration with respect to a kernel expressed in terms of special case of Meijer's $G$ function. An action of the regularized multiple Erdélyi-Kober operator on some simple kernels leads to decomposition formulas for the generalized hypergeometric functions. In the ultimate section, we define an alternative regularization better suited for representing the Bessel type generalized hypergeometric function ${ }_{p-1} F_{p}$. A particular case of this regularization is then used to identify some new facts about the positivity and reality of zeros of this function.
\end{abstract}

MSC 2010: Primary 26A33; Secondary 33C60, 33C20

Key Words and Phrases: multiple Erdélyi-Kober operator, Meijer's $G$ function, generalized hypergeometric function, Hadamard finite part

\section{Introduction: generalized fractional calculus}

Throughout the paper we will use the standard notation ${ }_{p} F_{q}$ for the generalized hypergeometric function (see [2, Section 2.1], [24, Section 5.1], [26. Sections 16.2-16.12] or [4, Chapter 12]) and $G_{p, q}^{m, n}$ for the Meijer's $G$ function (see [24, section 5.2], [26, 16.17], [27, 8.2] or [4, Chapter 12]).

In [20, Definition 1.1.1] V. Kiryakova introduced the generalized fractional calculus that emerged from the study of the hyper-Bessel integral

(c) Year Diogenes Co., Sofia

pp. $\mathrm{xxx}-\mathrm{xxx}$, DOI: 
and differential operators of Dimovski [23] and is based on the multiple Erdélyi-Kober operator defined for $\beta>0$ by

$$
\left[I_{\beta, p}^{\mathbf{a}, \mathbf{d}} f\right](x)=\int_{0}^{1} G_{0}(t) f\left(x t^{1 / \beta}\right) d t, \text { where } G_{0}(t):=G_{p, p}^{p, 0}\left(t \mid \begin{array}{l}
\mathbf{b}-1 \\
\mathbf{a}-1
\end{array}\right)
$$

and $\mathbf{d}=\mathbf{b}-\mathbf{a} \geq 0$ in the space $C_{\alpha}, \alpha \geq-\min (\mathbf{a})$, of continuous functions on $[0, \infty)$ satisfying the asymptotic relation $f(x)=O\left(x^{\alpha+\varepsilon}\right)$ as $x \rightarrow 0$ for any $\varepsilon>0$ (note a slight change of notation from [23]). See also more recent surveys [22] and [23]. One of the key results is the commutative factorization formula [20, Theorem 1.2.10]

$$
I_{\beta, p}^{\mathbf{a}, \mathbf{d}}=I_{\beta}^{a_{p}, d_{p}} \cdots I_{\beta}^{a_{2}, d_{2}} I_{\beta}^{a_{1}, d_{1}}
$$

in terms of Erdélyi-Kober operators [20, (1.1.17)]

$$
\left[I_{\beta}^{\alpha, \delta} f\right](x)=\frac{x^{-\beta(\alpha+\delta-1)}}{\Gamma(\delta)} \int_{0}^{x}\left(x^{\beta}-t^{\beta}\right)^{\delta-1} t^{\beta(\alpha-1)} f(t) d\left(t^{\beta}\right) .
$$

A further generalization with a kernel expressed in terms of Fox's $H$ function was considered in [22, (16)]. It was then shown in [20, Chapter 4] and 21] that all generalized hypergeometric functions can be expressed as the images of the operator $I_{\beta, p}^{\mathbf{a}, \mathbf{d}}$ acting on a few basic kernels, like exponentials, powers and (generalized) cosines. In our recent paper [11] as well in a series of papers [9, 10, 13, 15, 17, 18, by the first author jointly with Kalmykov, Prilepkina and Sitnik we demonstrated surprising effectiveness of such representations for discovering both new and known facts about the generalized hypergeometric functions. Furthermore, in [11] we suggested a simple method for regularizing the $\beta=1$ case of the integral 1.1 when it diverges (in particular, if $\alpha<-\min (\mathbf{a}))$. Writing $\phi_{x}(t): \rightarrow f(x t)$ in our formula [11, (39)] we obtain:

$$
\left[I_{1, p}^{\mathbf{a}, \mathbf{d}} f\right](x)=\sum_{k=0}^{n-1} \frac{(\mathbf{a})_{k} x^{k}}{(\mathbf{b})_{k} k !} f^{(k)}(0)+\frac{x^{n} \Gamma(\mathbf{b})}{\Gamma(\mathbf{a})} \int_{0}^{1} \widetilde{G}_{n}(t) f^{(n)}(x t) d t
$$

where $\mathbf{b}=\mathbf{a}+\mathbf{d}$ and

$$
\widetilde{G}_{n}(t):=G_{p+1, p+1}^{p+1,0}\left(t \mid \begin{array}{l}
\mathbf{b}-1+n, n \\
\mathbf{a}-1+n, 0
\end{array}\right), \quad n=0,1, \ldots
$$

This allows to extend the definition of $I_{1, p}^{\mathbf{a}, \mathbf{d}}$ to arbitrary complex parameters, but the price we pay is that $f$ has to be infinitely differentiable. As the kernels we act on in order to get the generalized hypergeometric functions satisfy a differentiability requirement, in [11] we obtained some representations of these functions that extend those from [20, Chapter 4] and [21]. 
In this paper, we continue this line of research and suggest a different regularization of the integral (1.1), which leads to another representation of the multiple Erdélyi-Kober operator $I_{\beta, p}^{\mathbf{a}, \mathbf{d}}$ involving derivatives of $f$ at the point $x$, see 2.10 and 3.19 below. This regularization can be applied for general $\beta>0$. More specifically, for certain values of the parameter vectors $\mathbf{a}=\left(a_{1}, \ldots, a_{p}\right)$ and $\mathbf{b}=\left(b_{1}, \ldots, b_{p}\right)$ the function $G_{0}(t)$ from 1.1 has non-integrable power singularities at $t=0$ and/or $t=1$. The regularization from [11, Section 5.2] uses the Taylor coefficients of the function $\varphi_{x}(t)=f(x t)$ at the point $t=0$ to overcome this divergence. The main emphasis in this work will be made on the regularization of (1.1) based on the expansion of $\varphi_{x}(t)$ in the neighborhood of $t=1$. This required a detailed investigation of the properties of the function $G_{p+1, p+1}^{p, 1}$ which are less known than those of the Meijer-Nørlund function $G_{p, p}^{p, 0}$ and might be of independent interest. We conduct such investigation in Section 2. In particular, we derive an identity relating $G_{p+1, p+1}^{p, 1}$ with $G_{p+1, p+1}^{p+1,0}$ (under a certain restriction on the parameters), a regularization formula for $G_{p+1, p+1}^{p, 1}$ when the poles of the integrand of different types superimpose, an expression for the moments of the function $G_{p+1, p+1}^{p, 1}$ and a formula for its hypergeometric transform which incorporates generalized Stieltjes, Laplace and Hankel transforms. Furthermore, we prove a proposition on sign stabilization for $G_{p+1, p+1}^{p, 1}$ when all but one of the parameters grow infinitely. In Section 3 we utilize the new properties from Section 2 to define and study a regularization of the integral (1.1) that uses Taylor coefficients of $\varphi_{x}(t)=f(x t)$ at the point $t=1$. Applying this regularization method to generalized Stieltjes, Laplace and cosine Fourier kernels we obtain new integral representations of the generalized hypergeometric functions. Finally, in the ultimate Section 4 we define an alternative regularization of (1.1) tailored for $\beta=2$ and better suited to serve the generalized hypergeometric function of the Bessel type. This approach leads to new information about positivity and real zeros of this function also presented in the same section.

\section{Regularization of the multiple Erdélyi-Kober operator}

Let us fix some notation and terminology first. The standard symbols $\mathbb{N}, \mathbb{Z}, \mathbb{R}$ and $\mathbb{C}$ will be used to denote the natural, integer, real and complex numbers, respectively; $\mathbb{N}_{0}=\mathbb{N} \cup\{0\}$. In what follows we will use the shorthand notation for the products and sums:

$$
\begin{gathered}
\Gamma(\mathbf{a}):=\Gamma\left(a_{1}\right) \Gamma\left(a_{2}\right) \cdots \Gamma\left(a_{p}\right),(\mathbf{a})_{n}:=\left(a_{1}\right)_{n}\left(a_{2}\right)_{n} \cdots\left(a_{p}\right)_{n}, \\
\mathbf{a}+\mu:=\left(a_{1}+\mu, a_{2}+\mu, \ldots, a_{p}+\mu\right) ;
\end{gathered}
$$


inequalities like $\Re(\mathbf{a})>0$ and properties like $-\mathbf{a} \notin \mathbb{N}_{0}$ will be understood element-wise (i.e. $-\mathbf{a} \notin \mathbb{N}_{0}$ means that no element of $\mathbf{a}$ is a non-positive integer). The key role in our construction will be played by the function

$$
G_{p+1, p+1}^{p, 1}\left(t \mid \begin{array}{l}
n, \mathbf{b}+n-1 \\
\mathbf{a}+n-1,0
\end{array}\right)=\frac{1}{2 \pi i} \int_{\mathcal{L}} \frac{\Gamma(\mathbf{a}+n-1+s) \Gamma(1-n-s)}{\Gamma(\mathbf{b}+n-1+s) \Gamma(1-s)} t^{-s} d s,
$$

which we denote $\widehat{G}_{n}(t)$, where $\mathbf{a}:=\left(a_{1}, \ldots, a_{p}\right), \mathbf{b}:=\left(b_{1}, \ldots, b_{p}\right)$ are (generally complex) parameter vectors. For $|t|<1$ the contour $\mathcal{L}$ is a left loop that separates the poles of the integrand of the form $a_{j l}=1-a_{j}-n-l$, $l \in \mathbb{N}_{0}$, leaving them on the left from the poles of the form $1-n+k, k \in \mathbb{N}_{0}$, leaving them on the right. By definition the two types of poles must not superimpose, which translates into the condition $-a_{j} \notin \mathbb{N}_{0}$. If they do, the definition can still be repaired by the regularization recently elaborated by us in [12, Proposition 2]. Further details regarding the choice of the contour and convergence of the integral can be found, for instance, in [19, section 1.1] or in [11, section 2].

Define $\mathcal{C B}^{\infty}[0,1]$ to be the class of functions on $[0,1]$ that have derivatives of all orders which are all bounded on $[0,1]$. If $\varphi \in \mathcal{C B}^{\infty}[0,1]$, then the integral 1.1 with $\varphi(t)=f\left(x t^{1 / \beta}\right)$ takes the form:

$$
\int_{0}^{1} G_{0}(t) \varphi(t) d t, \text { where } G_{0}(t)=G_{p, p}^{p, 0}\left(t \mid \begin{array}{l}
\mathbf{b}-1 \\
\mathbf{a}-1
\end{array}\right) .
$$

This integral converges (i.e. exists as an improper integral) if the next two conditions are satisfied:

$$
a:=\min \left(\Re a_{1}, \Re a_{2}, \ldots, \Re a_{p}\right)>0 \text { and } \Re(\psi):=\Re\left[\sum_{k=1}^{p}\left(b_{k}-a_{k}\right)\right]>0 .
$$

It also converges if the second condition is replaced by $\psi=0,-1,-2, \ldots$. These claims are immediate from the properties of $G_{0}(t)$ elaborated in [11, section 2]. Note that, for $\Re(\mathbf{a})>0$, the function $\widehat{G}_{n}(t)$ defined in 2.4 can be computed as the $n$-th primitive of $G_{0}(x)$ that satisfies $\widehat{G}_{n}^{(k)}(0)=0$ for $k=1,2, \ldots, n$

$$
\widehat{G}_{n}(t)=\frac{1}{(n-1) !} \int_{0}^{t} G_{0}(x)(t-x)^{n-1} d x .
$$

To convert the set $\mathcal{C B}^{\infty}[0,1]$ into a test function space, define the convergence in $\mathcal{C B}^{\infty}[0,1]$ as follows: a sequence $\left\{\varphi_{j}\right\} \subset \mathcal{C B}^{\infty}[0,1]$ converges to an element $\varphi \in \mathcal{C B}^{\infty}[0,1]$ if

$$
\max _{x \in[0,1]}\left|\varphi_{j}^{(k)}(x)-\varphi^{(k)}(x)\right| \rightarrow 0 \text { as } j \rightarrow \infty
$$


for each nonnegative integer $k$. This space can be viewed as a space of restrictions of smooth periodic functions (say with period 2) considered in [3, Chapter 3, paragraph 2] to the interval [0,1]. Then it follows from [3, Theorem 2.1] that this space is complete. In this section we will define a regularization of the integral $(2.5)$ assigning a finite value to it for arbitrary values of $a$ and $\psi$. Our regularization coincides with the analytic continuation in parameters $\mathbf{a}$ and $\mathbf{b}$, so that its application to the generalized Stieltjes, exponential or cosine Fourier kernel leads expectedly to the generalized hypergeometric functions.

Definition 2.1. For arbitrary complex $\mathbf{a}$ and $\mathbf{b},-\mathbf{b} \notin \mathbb{N}_{0}$, choose a nonnegative integer $n>-\min (a, \Re(\psi))$, where $a$ and $\psi$ are given in (2.6). Define a regularization of the integral (2.5) as the distribution $\mathcal{G}_{1}=\mathcal{G}_{1}(\mathbf{a}, \mathbf{b})$ acting on a test function $\varphi \in \mathcal{C B}^{\infty}[0,1]$ according to the formula

$$
\left\langle\mathcal{G}_{1}, \varphi\right\rangle=\sum_{k=0}^{n-1} \frac{(-1)^{k} \varphi^{(k)}(1)}{k !}{ }_{p+1} F_{p}\left(\begin{array}{c}
\mathbf{a},-k \\
\mathbf{b}
\end{array} \mid 1\right)+\frac{(-1)^{n} \Gamma(\mathbf{b})}{\Gamma(\mathbf{a})} \int_{0}^{1} \widehat{G}_{n}(t) \varphi^{(n)}(t) d t
$$

where $\widehat{G}_{n}(t)$ is given in 2.4 . If $n=0$ the finite sum in 2.8 is understood to be empty, so that 2.8 reduces to a multiple of 2.5 .

The asymptotic properties of $\widehat{G}_{n}(t)$ (at $t \rightarrow 0$ and $t \rightarrow 1$ ) contained in [12, Propositions 3 and 4] show the correctness of the above definition: the integral in $(2.8)$ exists as a finite number for all $\varphi \in \mathcal{C B}^{\infty}[0,1]$ under the conditions stated in the definition. When $n>0$ the above definition is motivated by the following argument. Replace $\varphi(t)$ in $(2.5)$ by its Taylor expansion at $t=1$ :

$$
\varphi(t)=\sum_{k=0}^{n-1} \frac{\varphi^{(k)}(1)}{k !}(t-1)^{k}+\varphi_{n}(t)
$$

where $\varphi_{n}(t)$ is the Taylor remainder. Applying [11, (19)], we obtain the right hand side of (2.8), but with the second term replaced by

$$
\frac{\Gamma(\mathbf{b})}{\Gamma(\mathbf{a})} \int_{0}^{1} G_{0}(t) \varphi_{n}(t) d t
$$

Integrating by parts $n$ times and using 2.7 and $\varphi_{n}^{(n)}(t)=\varphi^{(n)}(t), \varphi_{n}^{(k)}(1)=$ $\widehat{G}_{k+1}(0)=0$ for $k=0,1, \ldots, n-1$, we obtain 2.8 . Therefore, for any $n \in \mathbb{N}$ the integral (2.5) equals the right hand side of (2.8) when $a>0$ and $\Re(\psi)>0$. Moreover, the right hand side of $(2.8)$ is an analytic function of the parameters $\mathbf{a}$ and meromorphic function of the parameters $\mathbf{b}$ with 
simple poles at $-b_{i} \in \mathbb{N}_{0}$; hence, the right hand side of 2.8 gives an expression for the analytic continuation of $(2.5)$ in a to the domain $\Re(\mathbf{a})>$ $-n$ and its meromorphic continuation in $\mathbf{b}$ to the domain $\Re(\psi)>-n$. We conclude that the family of distributions $\mathcal{G}_{1}=\mathcal{G}_{1}(\mathbf{a}, \mathbf{b})$ is analytic in the parameters a and meromorphic in $\mathbf{b}$ with simple poles at $-b_{i} \in \mathbb{N}_{0}$ in the above domain.

Remark. The regularization defined in 2.8 can be easily seen to equal the Hadamard finite part of the divergent integral (2.5). See [7, 8] for details. However, we observe a new phenomenon here. In general, the Hadamard finite part constructed to overcome divergence at unity does not alter the situation at other points, while formula (2.8) regularizes the integral (2.5) at both points, 0 and 1, simultaneously.

Theorem 2.1. $\mathcal{G}_{1}$ is a continuous linear functional on $\mathcal{C B}^{\infty}[0,1]$ and its definition is independent of $n$.

$\mathrm{P}$ r o o f. Linearity is obvious. For continuity, assume that $\varphi_{j} \rightarrow \varphi$ in $\mathcal{C B}^{\infty}[0,1]$ and define $f_{k}:=(k !)^{-1}{ }_{p+1} F_{p}\left(\begin{array}{c}-k, \mathbf{a} \\ \mathbf{b}\end{array} \mid 1\right)$. Then

$$
\begin{aligned}
\mid\left\langle\mathcal{G}_{1}, \varphi_{j}\right\rangle & -\left\langle\mathcal{G}_{1}, \varphi\right\rangle|=|\left\langle\mathcal{G}_{1}, \varphi_{j}-\varphi\right\rangle\left|\leq \sum_{k=0}^{n-1}\right| f_{k}|| \varphi_{j}^{(k)}(1)-\varphi^{(k)}(1) \mid \\
& +\max _{x \in[0,1]}\left|\varphi_{j}^{(k)}(x)-\varphi^{(k)}(x)\right|\left|\frac{\Gamma(\mathbf{b})}{\Gamma(\mathbf{a})}\right| \int_{0}^{1}\left|\widehat{G}_{n}(t)\right| d t \rightarrow 0 \text { as } j \rightarrow \infty
\end{aligned}
$$

by the definition of convergence in $\mathcal{C B}^{\infty}[0,1]$ and because the last integral in finite by the asymptotic relations for $\widehat{G}_{n}(t)$ around $t=0$ and $t=1$ given in [12, Propositions 3 and 4]. Finally, write $\mathcal{G}_{1, n}$ for the distribution $\mathcal{G}_{1}$ with $n$ terms in the sum (2.8) and $\mathcal{G}_{1, m}$ for $m \neq n$ terms. By definition we must choose $n, m>-\Re(\psi)$. Assume without loss of generality that $n>m$ and let $\varphi$ be an arbitrary test function. Integration by parts yields

$$
\begin{aligned}
& \left\langle\mathcal{G}_{1, n}, \varphi\right\rangle-\left\langle\mathcal{G}_{1, m}, \varphi\right\rangle=\sum_{k=m}^{n-1}(-1)^{k} f_{k} \varphi^{(k)}(1) \\
& +\frac{(-1)^{n} \Gamma(\mathbf{b})}{\Gamma(\mathbf{a})} \int_{0}^{1} \widehat{G}_{n}(t) \varphi^{(n)}(t) d t-\frac{(-1)^{m} \Gamma(\mathbf{b})}{\Gamma(\mathbf{a})} \int_{0}^{1} \widehat{G}_{m}(t) \varphi^{(m)}(t) d t
\end{aligned}
$$




$$
\begin{aligned}
& =\sum_{k=m}^{n-1}(-1)^{k} f_{k} \varphi^{(k)}(1) \\
& +\frac{\Gamma(\mathbf{b})}{\Gamma(\mathbf{a})}\left\{(-1)^{n} \int_{0}^{1} \widehat{G}_{n}(t) \varphi^{(n)}(t) d t-\left.(-1)^{m} \widehat{G}_{m+1}(t) \varphi^{(m)}(t)\right|_{0} ^{1}\right. \\
& \left.\quad+(-1)^{m} \int_{0}^{1} \widehat{G}_{m+1}(t) \varphi^{(m+1)}(t) d t\right\} \\
& =\sum_{k=m+1}^{n-1}(-1)^{k} f_{k} \varphi^{(k)}(1)+\frac{(-1)^{n} \Gamma(\mathbf{b})}{\Gamma(\mathbf{a})} \int_{0}^{1} \widehat{G}_{n}(t) \varphi^{(n)}(t) d t \\
& \quad-\frac{(-1)^{m+1} \Gamma(\mathbf{b})}{\Gamma(\mathbf{a})} \int_{0}^{1} \widehat{G}_{m+1}(t) \varphi^{(m+1)}(t) d t
\end{aligned}
$$

where we used $\widehat{G}_{m+1}(0)=0, \widehat{G}_{m+1}(1)=\Gamma(\mathbf{a}) f_{m} / \Gamma(\mathbf{b})$ (see [12, Proposition 6] for the proof of the last formula). Repeating integration by parts $(n-m)$ times and using the above calculation clearly leads to $\left\langle\mathcal{G}_{1, n}, \varphi\right\rangle-$ $\left\langle\mathcal{G}_{1, m}, \varphi\right\rangle=0$.

We can now apply regularization (2.8) to extend the definition of the multiple Erdélyi-Kober operator (1.1). Taking

$$
\phi_{x}(t)=f\left(x t^{1 / \beta}\right)
$$

we can calculate, using [6, Exercise 7, p.157],

$$
\frac{d^{k}}{d t^{k}} \phi_{x}(t)=\sum_{j=1}^{k} f^{(j)}\left(x t^{1 / \beta}\right) x^{j} t^{j / \beta-k} Z_{k, j}(1 / \beta),
$$

with coefficients $Z_{k, j}(\alpha)$ generated by

$$
\frac{\left((1+y)^{\alpha}-1\right)^{j}}{j !}=\sum_{k \geq j} Z_{k, j}(\alpha) \frac{y^{k}}{k !} .
$$

Substituting these formulas into 2.8 we obtain

$$
\begin{array}{r}
{\left[I_{\beta, p}^{\mathbf{a}, \mathbf{d}} f\right](x)=\sum_{k=0}^{n-1} \frac{(-1)^{k}}{k !}{ }_{p+1} F_{p}\left(\begin{array}{c}
\mathbf{a},-k \\
\mathbf{b}
\end{array} \mid 1\right) \sum_{j=1}^{k} f^{(j)}(x) x^{j} Z_{k, j}(1 / \beta)} \\
+\frac{(-1)^{n} \Gamma(\mathbf{b})}{\Gamma(\mathbf{a})} \sum_{j=1}^{n} x^{j} Z_{n, j}(1 / \beta) \int_{0}^{1} \widehat{G}_{n}(t) f^{(j)}\left(x t^{1 / \beta}\right) t^{j / \beta-n} d t
\end{array}
$$


As, clearly, $Z_{k, j}(1)=0$ for $j=1, \ldots, k-1$ and $Z_{k, k}(1)=1$, the above formula simplifies for $\beta=1$ to

$$
\begin{aligned}
{\left[I_{\beta, p}^{\mathbf{a}, \mathbf{d}} f\right](x)=\sum_{k=0}^{n-1} \frac{(-x)^{k}}{k !} p+1 } & F_{p}\left(\begin{array}{c}
\mathbf{a},-k \\
\mathbf{b}
\end{array} \mid 1\right) f^{(k)}(x) \\
& +\frac{(-x)^{n} \Gamma(\mathbf{b})}{\Gamma(\mathbf{a})} \int_{0}^{1} \widehat{G}_{n}(t) f^{(n)}(x t) d t .
\end{aligned}
$$

Note that the coefficients $Z_{k, j}(\alpha)$ can be computed explicitly for $\alpha=1 / 2$ and $\alpha=2$ by [6, Exercise 7, p.157]:

$$
\begin{gathered}
Z_{k, j}(1 / 2)=(-1)^{k-j} \frac{(k-1) !}{(j-1) !}\left(\begin{array}{c}
2 k-j-1 \\
k-1
\end{array}\right) \frac{1}{2^{2 k-j}} \\
Z_{k, j}(2)=\frac{k !}{j !}\left(\begin{array}{c}
j \\
k-j
\end{array}\right) \frac{1}{2^{2 j-k}}
\end{gathered}
$$

Theorem 2.2. For arbitrary $\mathbf{a} \in \mathbb{C}^{p}, \mathbf{b} \in \mathbb{C}^{p},-\mathbf{b} \notin \mathbb{N}_{0}$, and $\mathbf{c} \in \mathbb{C}^{u}$, $\mathbf{d} \in \mathbb{C}^{s},-\mathbf{d} \notin \mathbb{N}_{0}$, choose a nonnegative integer $n>-\min (a, \Re(\psi))$, where $a$ and $\psi$ are defined in (2.6). Then

$$
\left\langle\mathcal{G}_{1}(\mathbf{a}, \mathbf{b}),{ }_{u} F_{s}(\mathbf{c} ; \mathbf{d} ;-z t)\right\rangle={ }_{u+p} F_{s+p}\left(\begin{array}{c}
\mathbf{a}, \mathbf{c} \\
\mathbf{b}, \mathbf{d}
\end{array} \mid-z\right)
$$

for all $z \in \mathbb{C}$ if $u \leq s$ and for all $z \in \mathbb{C} \backslash(-\infty,-1]$ if $u=s+1$.

P r o o f. Indeed, for $\Re(\psi)>0$ and $\Re(\mathbf{a})>0$ the action of $\mathcal{G}_{1}$ reduces to the integral (2.5), so that formula 2.11) coincides with [10, (4)]. For general a and $\psi$ the claim then follows by analytic continuation in parameters, as both sides of (2.11) are analytic in $\mathbf{a}$ and meromorphic in $\mathbf{b}$.

Remark. By definition of $\mathcal{G}_{1}$ the expression on the left hand side of (2.11) is the sum

$$
\begin{aligned}
\sum_{k=0}^{n-1} \frac{(\mathbf{c})_{k} z^{k}}{(\mathbf{d})_{k} k !}{ }_{u} F_{s}(\mathbf{c}+k ; \mathbf{d} & +k ;-z)_{p+1} F_{p}\left(\begin{array}{c}
\mathbf{a},-k \\
\mathbf{b}
\end{array} \mid 1\right) \\
& +\frac{z^{n}(\mathbf{c})_{n} \Gamma(\mathbf{b})}{(\mathbf{d})_{n} \Gamma(\mathbf{a})} \int_{0}^{1} \widehat{G}_{n}(t)_{u} F_{s}(\mathbf{c}+n ; \mathbf{d}+n ;-z t) d t
\end{aligned}
$$

The fact that this sum is equal to the generalized hypergeometric function of the right hand side of (2.11) can also be established directly by a combination of [12, Proposition 7] and [12, Corollary 3]. This gives an alternative direct proof of the above theorem. 
The most important particular cases of Theorem 2.2 are given in the next corollary.

Corollary 2.1. Under conditions of Theorem 2.2 we have:

$$
\left\langle\mathcal{G}_{1}(\mathbf{a}, \mathbf{b}),(1+z t)^{-\sigma}\right\rangle={ }_{p+1} F_{p}\left(\begin{array}{c}
\sigma, \mathbf{a} \\
\mathbf{b}
\end{array} \mid-z\right)
$$

for all $z \in \mathbb{C} \backslash(-\infty,-1], \sigma \in \mathbb{C}$,

$$
\left\langle\mathcal{G}_{1}(\mathbf{a}, \mathbf{b}), e^{-z t}\right\rangle={ }_{p} F_{p}\left(\begin{array}{l}
\mathbf{a} \\
\mathbf{b}
\end{array} \mid-z\right)
$$

and

$$
\left\langle\mathcal{G}_{1}(\mathbf{a}, \mathbf{b}), \cos (2 \sqrt{z t})\right\rangle={ }_{p-1} F_{p}\left(\begin{array}{l}
\hat{\mathbf{a}} \\
\mathbf{b}
\end{array} \mid-z\right)
$$

for all $z \in \mathbb{C}$, where $\mathbf{a}=(\hat{\mathbf{a}}, 1 / 2)$ in the last formula.

P r o o f. Indeed, (2.12) and (2.13) are obviously particular cases of (2.11). The last formula (2.14) is also a particular case of (2.11) in view of $\mathbf{a}=(\hat{\mathbf{a}}, 1 / 2)$ and $\cos (2 \sqrt{z t})={ }_{0} F_{1}(-; 1 / 2 ;-z t)$.

The next elementary lemma on sign stabilization of the Riemann-Liouville fractional integral may be of independent interest. The proof is given in [12, Lemma 1].

Lemma 2.1. Suppose $f:(0,1] \rightarrow \mathbb{R}$ is continuous and integrable (possibly in improper sense). If $f>0$ in some neighborhood of zero, then there exists $\alpha>0$ such that the Riemann-Liouville fractional integral

$$
\left[I_{+}^{\alpha} f\right](x)=\frac{1}{\Gamma(\alpha)} \int_{0}^{x} f(t)(x-t)^{\alpha-1} d t
$$

is positive for all $x \in(0,1]$.

The above lemma leads to the following statement regarding the sign stabilization of $\widehat{G}_{n} / \Gamma(\mathbf{a})$ as $n$ grows to infinity.

Corollary 2.2. For arbitrary real vectors $\mathbf{a}$, b there exists $N \in \mathbb{N}_{0}$ such that

$$
\frac{(-1)^{\eta}}{\Gamma(\mathbf{a})} G_{p+1, p+1}^{p, 1}\left(t \mid \begin{array}{l}
n, \mathbf{b}+n-1 \\
\mathbf{a}+n-1,0
\end{array}\right)>0
$$

for all $n \geq N, t \in(0,1]$, where $\eta \in\{0,1\}$ is chosen so that this expression is positive in arbitrarily small right neighborhood of $t=0$. 
Remark. If $\mathbf{b}-a$ does not contain non-positive integers, where $a$ is defined in (2.6), then

$$
(-1)^{\eta}=\operatorname{sgn}\left[\frac{1}{\Gamma(\mathbf{a})(a)_{n} \prod_{i=1}^{p} \Gamma\left(b_{i}-a\right)}\right] .
$$

Further details on determination of $\eta$ are given in [12, (22)].

The combination of Corollary 2.1 with Corollary 2.2 leads to the decomposition formulas presented in the following theorem.

Theorem 2.3. Suppose that $\mathbf{a}, \mathbf{b}$ are arbitrary real vectors of size $p$ such that $-\mathbf{b} \notin \mathbb{N}_{0}$ and $\sigma$ is any real number. Then there exists $N \in \mathbb{N}_{0}$, such that for all $n \geq N$,

$$
\begin{gathered}
\frac{1}{\Gamma(\mathbf{b})} p+1 F_{p}\left(\begin{array}{c}
\sigma, \mathbf{a} \\
\mathbf{b}
\end{array} \mid-z\right)=\frac{1}{\Gamma(\mathbf{b})} \sum_{k=0}^{n-1} \frac{z^{k}(\sigma)_{k}}{(z+1)^{\sigma+k} k !} p+1 F_{p}\left(\begin{array}{c}
\mathbf{a},-k \\
\mathbf{b}
\end{array}\right) \\
+(-1)^{\eta}(\sigma)_{n} z^{n} \int_{0}^{1} \frac{\mu_{n}(d t)}{(1+z t)^{\sigma+n}} \\
\frac{1}{\Gamma(\mathbf{b})}{ }_{p} F_{p}\left(\begin{array}{c}
\mathbf{a} \\
\mathbf{b}
\end{array} \mid-z\right)=\frac{e^{-z}}{\Gamma(\mathbf{b})} \sum_{k=0}^{n-1} \frac{z^{k}}{k !} p+1 F_{p}\left(\begin{array}{c}
\mathbf{a},-k \\
\mathbf{b}
\end{array} \mid 1\right)+(-1)^{\eta} z^{n} \int_{0}^{1} e^{-z t} \mu_{n}(d t)
\end{gathered}
$$

and, with $\mathbf{a}=(\hat{\mathbf{a}}, 1 / 2)$,

$$
\begin{aligned}
\frac{1}{\Gamma(\mathbf{b})}{ }_{p-1} F_{p}\left(\begin{array}{c}
\hat{\mathbf{a}} \\
\mathbf{b}
\end{array} \mid-z\right)= & \frac{1}{\Gamma(\mathbf{b})} \sum_{k=0}^{n-1} \frac{z^{k}{ }_{0} F_{1}(-; k+1 / 2 ;-z)}{(1 / 2)_{k} k !}{ }_{p+1} F_{p}\left(\begin{array}{c}
-k, \mathbf{a} \\
\mathbf{b}
\end{array} \mid 1\right) \\
& +(-1)^{\eta} \frac{z^{n}}{(1 / 2)_{n}} \int_{0}^{1}{ }_{0} F_{1}(-; n+1 / 2 ;-z t) \mu_{n}(d t),
\end{aligned}
$$

where

$$
\mu_{n}(d t):=\frac{(-1)^{\eta}}{\Gamma(\mathbf{a})} G_{p+1, p+1}^{p, 1}\left(t \mid \begin{array}{l}
n, \mathbf{b}+n-1 \\
\mathbf{a}+n-1,0
\end{array}\right) d t
$$

is a positive measure. The meaning of $\eta$ is explained in Corollary 2.2 .

\section{Alternative regularization for $\beta=2$}

The regularized expression 2.10 is rather complicated even for $\beta=2$ when the coefficients $Z_{k, j}(1 / 2)$ are given explicitly. In this section we suggest an alternative regularization for $\beta=2$ which corresponds to a composition of the classical Kober-Erdélyi operators. This new regularization does not involve the coefficients $Z_{k, j}$ and avoids double sums. Furthermore, the decomposition corresponding to $\left\langle\mathcal{G}_{1}(\mathbf{a}, \mathbf{b}), \cos (\sqrt{z t})\right\rangle$ given by the last formula of Theorem 2.3 contains a non-elementary Bessel function ${ }_{0} F_{1}$. Our 
alternative regularization developed in this section contains only the elementary cosine function, which is useful in particular, when studying zeros of the Bessel type hypergeometric function. It is natural to start with [11, $(5)]$ :

$$
{ }_{p-1} F_{p}\left(\begin{array}{l}
\hat{\mathbf{a}} \\
\mathbf{b}
\end{array} \mid-z\right)=\frac{\Gamma(\mathbf{b})}{\sqrt{\pi} \Gamma(\hat{\mathbf{a}})} \int_{0}^{1} \cos (2 \sqrt{z t}) G_{p, p}^{p, 0}\left(t \mid \begin{array}{l}
\mathbf{b}-1 \\
\hat{\mathbf{a}}-1,-1 / 2
\end{array}\right) d t .
$$

Setting $t=u^{2}$ and changing $z \rightarrow z^{2} / 4$ we get

$$
{ }_{p-1} F_{p}\left(\begin{array}{c}
\hat{\mathbf{a}} \\
\mathbf{b}
\end{array} \mid-\frac{z^{2}}{4}\right)=\frac{2 \Gamma(\mathbf{b})}{\sqrt{\pi} \Gamma(\hat{\mathbf{a}})} \int_{0}^{1} \cos (z u) G_{p, p}^{p, 0}\left(\begin{array}{l|l}
u^{2} & \begin{array}{l}
\mathbf{b}-1 / 2 \\
\hat{\mathbf{a}}-1 / 2,0
\end{array}
\end{array}\right) d u .
$$

Hence, we need to regularize integrals of the form

$$
\int_{0}^{1} G_{p, p}^{p, 0}\left(u^{2} \mid \begin{array}{l}
\mathbf{b}-1 / 2 \\
\mathbf{a}-1 / 2
\end{array}\right) \phi(u) d u=\frac{1}{2} \int_{0}^{1} G_{p, p}^{p, 0}\left(t \mid \begin{array}{l}
\mathbf{b}-1 \\
\mathbf{a}-1
\end{array}\right) \phi(\sqrt{t}) d t
$$

which corresponds to the regularization of the multiple Erdélyi-Kober operator 1.1) with $\beta=2$ once we write $\phi_{x}(\sqrt{t})=f(x \sqrt{t})$. Assuming $\phi \in \mathcal{C B}^{\infty}[0,1]$, we apply Taylor's theorem with integral remainder to $\phi$ in the neighborhood of $u=1$ to obtain

$$
\begin{gathered}
\int_{0}^{1} G_{p, p}^{p, 0}\left(u^{2} \mid \begin{array}{l}
\mathbf{b}-1 / 2 \\
\mathbf{a}-1 / 2
\end{array}\right) \phi(u) d u=\int_{0}^{1} G_{p, p}^{p, 0}\left(u^{2} \mid \begin{array}{l}
\mathbf{b}-1 / 2 \\
\mathbf{a}-1 / 2
\end{array}\right) \\
\times\left[\sum_{k=0}^{n-1} \frac{\phi^{(k)}(1)}{k !}(u-1)^{k}+\frac{1}{(n-1) !} \int_{1}^{u}(u-t)^{n-1} \phi^{(n)}(t) d t\right] d u \\
=\sum_{k=0}^{n-1} \frac{\phi^{(k)}(1)}{(-1)^{k} k !} \int_{0}^{1} G_{p, p}^{p, 0}\left(u^{2} \mid \begin{array}{l}
\mathbf{b}-1 / 2 \\
\mathbf{a}-1 / 2
\end{array}\right)(1-u)^{k} d u \\
+\frac{(-1)^{n}}{\Gamma(n)} \int_{0}^{1} G_{p, p}^{p, 0}\left(u^{2} \mid \begin{array}{l}
\mathbf{b}-1 / 2 \\
\mathbf{a}-1 / 2
\end{array}\right) d u \int_{u}^{1}(t-u)^{n-1} \phi^{(n)}(t) d t .
\end{gathered}
$$

By substitution $t=u^{2}$ and separation of odd and even terms, the leftmost integral in the last expression is elaborated as follows:

$$
\begin{aligned}
& \int_{0}^{1} G_{p, p}^{p, 0}\left(u^{2} \mid \begin{array}{l}
\mathbf{b}-1 / 2 \\
\mathbf{a}-1 / 2
\end{array}\right)(1-u)^{k} d u=\sum_{j=0}^{k}(-1)^{j}\left(\begin{array}{c}
k \\
j
\end{array}\right) \int_{0}^{1} G_{p, p}^{p, 0}\left(u^{2} \mid \begin{array}{l}
\mathbf{b}-1 / 2 \\
\mathbf{a}-1 / 2
\end{array}\right) u^{j} d u \\
= & \frac{1}{2} \sum_{j=0}^{k}(-1)^{j}\left(\begin{array}{l}
k \\
j
\end{array}\right) \int_{0}^{1} G_{p, p}^{p, 0}\left(t \mid \begin{array}{l}
\mathbf{b}-1 / 2 \\
\mathbf{a}-1 / 2
\end{array}\right) t^{j / 2-1 / 2} d t=\sum_{j=0}^{k} \frac{(-k)_{j} \Gamma(\mathbf{a}+j / 2)}{2 \Gamma(\mathbf{b}+j / 2) j !}
\end{aligned}
$$




$$
\begin{gathered}
=\sum_{\substack{j=0 \\
j=2 m}}^{k} \frac{(-k)_{j} \Gamma(\mathbf{a}+j / 2)}{2 \Gamma(\mathbf{b}+j / 2) j !}+\sum_{\substack{j=0 \\
j=2 m+1}}^{k} \frac{(-k)_{j} \Gamma(\mathbf{a}+j / 2)}{2 \Gamma(\mathbf{b}+j / 2) j !} \\
=\sum_{m=0}^{k / 2} \frac{(-k / 2)_{m}(-k / 2+1 / 2)_{m} \Gamma(\mathbf{a}+m)}{2(1 / 2)_{m} \Gamma(\mathbf{b}+m) m !} \\
+\sum_{m=0}^{(k-1) / 2} \frac{(-k)(-k / 2+1)_{m}(-k / 2+1 / 2)_{m} \Gamma(\mathbf{a}+1 / 2+m)}{2 \Gamma(\mathbf{b}+1 / 2+m)(3 / 2)_{m} m !} \\
=\frac{\Gamma(\mathbf{a})}{2 \Gamma(\mathbf{b})} p+2 F_{p+1}\left(\begin{array}{c}
-k / 2,-k / 2+1 / 2, \mathbf{a} \\
1 / 2, \mathbf{b}
\end{array}\right) \\
-\frac{k \Gamma(\mathbf{a}+1 / 2)}{2 \Gamma(\mathbf{b}+1 / 2)} p+2 F_{p+1}\left(\begin{array}{c}
-k / 2+1,-k / 2+1 / 2, \mathbf{a}+1 / 2 \\
3 / 2, \mathbf{b}+1 / 2
\end{array}\right),
\end{gathered}
$$

where we utilized the shorthand notation ${ }_{p} F_{q}(\mathbf{a} ; \mathbf{b})={ }_{p} F_{q}(\mathbf{a} ; \mathbf{b} ; 1)$ and the easily verifiable identities

$$
\begin{aligned}
& (2 m) !=4^{m}(1 / 2)_{m} m !,(2 m+1) !=4^{m}(3 / 2)_{m} m ! \\
& (-k)_{2 m}=4^{m}(-k / 2)_{m}(-k / 2+1 / 2)_{m}, \\
& (-k)_{2 m+1}=(-k) 4^{m}(-k / 2+1)_{m}(-k / 2+1 / 2)_{m} .
\end{aligned}
$$

Further, using [27, formula 2.24.2.2] for the second term in the last line of (3.17), we get

$$
\begin{aligned}
& \int_{0}^{1} G_{p, p}^{p, 0}\left(u^{2} \mid \begin{array}{l}
\mathbf{b}-1 / 2 \\
\mathbf{a}-1 / 2
\end{array}\right) d u \int_{u}^{1}(t-u)^{n-1} \phi^{(n)}(t) d t \\
& =\int_{0}^{1} \phi^{(n)}(t) d t \int_{0}^{t} G_{p, p}^{p, 0}\left(u^{2} \mid \begin{array}{l}
\mathbf{b}-1 / 2 \\
\mathbf{a}-1 / 2
\end{array}\right)(t-u)^{n-1} d u \\
& =\frac{(n-1) !}{2^{n}} \int_{0}^{1} G_{p+2, p+2}^{p, 2}\left(t^{2} \mid \begin{array}{l}
n / 2,(n+1) / 2, \mathbf{b}+(n-1) / 2 \\
\mathbf{a}+(n-1) / 2,0,1 / 2
\end{array}\right) \phi^{(n)}(t) d t .
\end{aligned}
$$

Combining these formulas we can define the regularization of the integral (3.16) as follows.

Definition 3.1. For arbitrary complex $\mathbf{a}$ and $\mathbf{b},-\mathbf{b} \notin \mathbb{N}_{0}$, choose a nonnegative integer $n>-\min (a, \Re(\psi))$, where $a$ and $\psi$ are given in (2.6). Define a regularization of the integral $(3.16)$ as the distribution $\mathcal{G}_{b}^{1}=\mathcal{G}_{b}^{1}(\mathbf{a}, \mathbf{b})$ acting on a test function $\phi \in \mathcal{C B}^{\infty}[0,1]$ according to the 
formula

$$
\begin{aligned}
\left\langle\mathcal{G}_{b}^{1}, \phi\right\rangle & =\sum_{k=0}^{n-1} \frac{\phi^{(k)}(1)}{(-1)^{k} k !}\left\{\frac{\Gamma(\mathbf{a})}{2 \Gamma(\mathbf{b})}{ }_{p+2} F_{p+1}\left(\begin{array}{c}
-k / 2,-k / 2+1 / 2, \mathbf{a} \\
1 / 2, \mathbf{b}
\end{array}\right)\right. \\
& \left.-\frac{k \Gamma(\mathbf{a}+1 / 2)}{2 \Gamma(\mathbf{b}+1 / 2)} p+2 F_{p+1}\left(\begin{array}{c}
-k / 2+1,-k / 2+1 / 2, \mathbf{a}+1 / 2 \\
3 / 2, \mathbf{b}+1 / 2
\end{array}\right)\right\} \\
+ & \frac{(-1)^{n}}{2^{n}} \int_{0}^{1} G_{p+2, p+2}^{p, 2}\left(\begin{array}{l|l}
t^{2} & \begin{array}{l}
n / 2,(n+1) / 2, \mathbf{b}+(n-1) / 2 \\
\mathbf{a}+(n-1) / 2,0,1 / 2
\end{array}
\end{array}\right) \phi^{(n)}(t) d t .
\end{aligned}
$$

If $n=0$ the finite sum in (3.18) is understood to be empty, so that (3.18) reduces to a multiple of $(3.16)$.

An argument similar to that given in the previous section shows that $\mathcal{G}_{b}^{1}$ is a continuous linear functional on $\mathcal{C B}^{\infty}[0,1]$, whose definition is independent of $n$. Furthermore, $\left\langle\mathcal{G}_{b}^{1}, \phi\right\rangle$ coincides with the analytic continuation of (3.16) in the parameters $\mathbf{a}$ and $\mathbf{b}$. In view of (3.16) choosing $\phi_{x}(u)=f(x u)=f(x \sqrt{t})$ we obtain a regularization of the multiple ErdélyiKober operator 1.1 with $\beta=2$ as follows:

$$
\begin{gathered}
{\left[I_{2, p}^{\mathbf{a}, \mathbf{d}} f\right](x)=2 \sum_{k=0}^{n-1} \frac{x^{k} f^{(k)}(x)}{(-1)^{k} k !}\left\{\frac{\Gamma(\mathbf{a})}{2 \Gamma(\mathbf{b})} p+2 F_{p+1}\left(\begin{array}{c}
-k / 2,-k / 2+1 / 2, \mathbf{a} \\
1 / 2, \mathbf{b}
\end{array}\right)\right.} \\
\left.-\frac{k \Gamma(\mathbf{a}+1 / 2)}{2 \Gamma(\mathbf{b}+1 / 2)} p+2 F_{p+1}\left(\begin{array}{c}
-k / 2+1,-k / 2+1 / 2, \mathbf{a}+1 / 2 \\
3 / 2, \mathbf{b}+1 / 2
\end{array}\right)\right\} \\
+\frac{(-x)^{n}}{2^{n-1}} \int_{0}^{1} G_{p+2, p+2}^{p, 2}\left(t^{2} \mid \begin{array}{l}
n / 2,(n+1) / 2, \mathbf{b}+(n-1) / 2 \\
\mathbf{a}+(n-1) / 2,0,1 / 2
\end{array}\right) f^{(n)}(x t) d t
\end{gathered}
$$

Using

$$
\frac{\partial^{k}}{\partial u^{k}} \cos (z u)=z^{k} \cos (z u+\pi k / 2)
$$


and setting $\mathbf{a}=(\hat{\mathbf{a}}, 1 / 2)$, we then obtain

$$
\begin{aligned}
& { }_{p-1} F_{p}\left(\begin{array}{l}
\hat{\mathbf{a}} \\
\mathbf{b}
\end{array} \mid-\frac{z^{2}}{4}\right)=\sum_{k=0}^{n-1} \frac{z^{k} \cos (z+\pi k / 2)}{(-1)^{k} k !}\left\{\begin{array}{l}
{ }_{p+2} F_{p+1}\left(\begin{array}{c}
-k / 2,-k / 2+1 / 2, \mathbf{a} \\
1 / 2, \mathbf{b}
\end{array}\right) \\
\left.-k \frac{\Gamma(\mathbf{b}) \Gamma(\mathbf{a}+1 / 2)}{\Gamma(\mathbf{a}) \Gamma(\mathbf{b}+1 / 2)} p_{p+2} F_{p+1}\left(\begin{array}{c}
-k / 2+1,-k / 2+1 / 2, \mathbf{a}+1 / 2 \\
3 / 2, \mathbf{b}+1 / 2
\end{array}\right)\right\}+ \\
\frac{(-z)^{n} \Gamma(\mathbf{b})}{2^{n-1} \Gamma(\mathbf{a})} \int_{0}^{1} G_{p+2, p+2}^{p, 2}\left(t^{2} \mid \begin{array}{l}
n / 2,(n+1) / 2, \mathbf{b}+(n-1) / 2 \\
\mathbf{a}+(n-1) / 2,0,1 / 2
\end{array}\right) \cos (z t+\pi n / 2) d t .
\end{array}\right.
\end{aligned}
$$

We will use a particular case of (3.20) to extract some information about the zeros of the function on the left hand side. In order to do his, we need to recall some facts regarding the positivity of the Meijer-Nørlund function $G_{p, p}^{p, 0}$. We follow [11, Property 9]. The inequality

$$
G_{p, p}^{p, 0}\left(\begin{array}{l|l}
x & \mathbf{b} \\
\mathbf{a}
\end{array}\right) \geq 0 \text { for } 0<x<1
$$

holds if $v_{\mathbf{a}, \mathbf{b}}(t):=\sum_{j=1}^{p}\left(t^{a_{j}}-t^{b_{j}}\right) \geq 0$ for $t \in[0,1]$. See [10, Theorem 2] for a proof of this fact and [14, Section 2] for further details. Note also that $v_{\mathbf{a}, \mathbf{b}}(t) \geq 0$ implies that $\psi=\sum_{j=1}^{p}\left(b_{j}-a_{j}\right) \geq 0$. For given $\mathbf{a}, \mathbf{b}$ the inequality $v_{\mathbf{a}, \mathbf{b}}(t) \geq 0$ is not easy to verify other than numerically. However, several sufficient conditions for $v_{\mathbf{a}, \mathbf{b}}(t) \geq 0$ expressed directly in terms of $\mathbf{a}, \mathbf{b}$ are known. In particular, according to [1, Theorem 10] $v_{\mathbf{a}, \mathbf{b}}(t) \geq 0$ on $[0,1]$ if

$$
\begin{aligned}
& 0<a_{1} \leq a_{2} \leq \cdots \leq a_{p}, 0<b_{1} \leq b_{2} \leq \cdots \leq b_{p}, \\
& \sum_{i=1}^{k} a_{i} \leq \sum_{i=1}^{k} b_{i} \text { for } k=1,2 \ldots, p .
\end{aligned}
$$

These inequalities are known as the weak supermajorization and are abbreviated as $\mathbf{b} \prec^{W} \mathbf{a}$. Further sufficient conditions can be found in [11, Property 9] and [14, Section 2].

Lemma 3.1. Suppose $\alpha \geq 0, \beta-\alpha \geq 1$, $\mathbf{a}>\beta-1$ and $v_{\mathbf{a}, \mathbf{b}}(t) \geq 0$ on $[0,1]$ (in particular, $\mathbf{b}-\beta+1 \prec^{W} \mathbf{a}-\beta+1$ is sufficient). Then the function

$$
x \rightarrow G_{p+1, p+1}^{p, 1}\left(x \mid \begin{array}{l}
\beta, \mathbf{b} \\
\mathbf{a}, \alpha
\end{array}\right)
$$

is positive and increasing on $(0,1)$. 
P r o o f. Set $\gamma:=\beta-\alpha \geq 1, \mathbf{b}^{\prime}:=\mathbf{b}-\beta, \mathbf{a}^{\prime}:=\mathbf{a}-\beta$. We have

$$
\begin{aligned}
G_{p+1, p+1}^{p, 1}\left(x \mid \begin{array}{l}
\beta, \mathbf{b} \\
\mathbf{a}, \alpha
\end{array}\right)=x^{\alpha} G_{p+1, p+1}^{p, 1} & \left(x \mid \begin{array}{c}
\gamma, \mathbf{b}^{\prime}+\gamma \\
\mathbf{a}^{\prime}+\gamma, 0
\end{array}\right) \\
= & \frac{x^{\alpha}}{\Gamma(\gamma)} \int_{0}^{x}(x-t)^{\gamma-1} G_{p, p}^{p, 0}\left(t \mid \begin{array}{l}
\mathbf{b}^{\prime} \\
\mathbf{a}^{\prime}
\end{array}\right) d t
\end{aligned}
$$

according to [27, 2.24.2.2]. The nonnegativity of the $G$ function in the integrand combined with $\gamma-1 \geq 0$ completes the proof.

Theorem 3.1. Let $\hat{\mathbf{a}}, \mathbf{b}$ be positive vectors. Set $\mathbf{a}=(\hat{\mathbf{a}}, 1 / 2)$ and assume that $v_{\mathbf{a}, \mathbf{b}}(t) \geq 0$ on $[0,1]$ (in particular, $\mathbf{b} \prec^{W} \mathbf{a}$ is sufficient). Then all zeros of

$$
f(z)={ }_{p-1} F_{p}\left(\begin{array}{l}
\hat{\mathbf{a}} \\
\mathbf{b}
\end{array} \mid-\frac{z^{2}}{4}\right)-\cos (z)
$$

are real and simple. Each interval $(\pi, 2 \pi),(2 \pi, 3 \pi)$ contains exactly one zero of $f$ and there are no other zeros except the trivial one at $z=0$.

P r o o f. Using (3.20) for $n=1$ we obtain

$$
{ }_{p-1} F_{p}\left(\begin{array}{l}
\hat{\mathbf{a}} \\
\mathbf{b}
\end{array} \mid-\frac{z^{2}}{4}\right)=\cos (z)+\frac{z \Gamma(\mathbf{b})}{\Gamma(\mathbf{a})} \int_{0}^{1} G_{p+1, p+1}^{p, 1}\left(t^{2} \mid \begin{array}{l}
1, \mathbf{b} \\
\mathbf{a}, 0
\end{array}\right) \sin (z t) d t,
$$

where $\mathbf{a}=(\hat{\mathbf{a}}, 1 / 2)$. According to Lemma 3.1 the $G$ function in the integrand is positive and increasing and is obviously not a step function. The claim now follows by [28, Theorem 2.2.2].

In a nice recent paper [5] the authors found the exact range of positive parameters $\alpha, \beta_{1}, \beta_{2}$ that ensure the inequality ${ }_{1} F_{2}\left(\alpha ; \beta_{1}, \beta_{2} ; x\right) \geq 0$ for all real $x$. This range can be described as follows: for $\alpha>0$ let $P_{\alpha}$ denote the convex hull of the points $\left(\alpha_{m}, \infty\right),\left(\alpha_{m}, \alpha_{M}\right),\left(\alpha_{M}, \alpha_{m}\right),\left(\infty, \alpha_{m}\right)$ in the plane $\left(\beta_{1}, \beta_{2}\right)$, where $\alpha_{m}=\min (2 \alpha, \alpha+1 / 2), \alpha_{M}=\max (2 \alpha, \alpha+$ $1 / 2)$. Then ${ }_{1} F_{2}\left(\alpha ; \beta_{1}, \beta_{2} ; x\right) \geq 0$ for $\alpha, \beta_{1}, \beta_{2}>0$ iff $\left(\beta_{1}, \beta_{2}\right) \in P_{\alpha}$. In the final section the authors extend their results to the Bessel type generalized hypergeometric function ${ }_{p-1} F_{p}$. Their extension theorem can be strengthen as follows.

Theorem 3.2. Suppose that $\alpha>0,\left(\beta_{1}, \beta_{2}\right) \in P_{\alpha}, \mathbf{a}, \mathbf{b} \in \mathbb{R}^{p-1}$ with $\mathbf{a}>0$ and $v_{\mathbf{a}, \mathbf{b}}(t) \geq 0$ on $[0,1]$ (in particular, $\mathbf{b} \prec^{W} \mathbf{a}$ is sufficient). Then

$$
{ }_{p} F_{p+1}\left(\begin{array}{c}
\alpha, \mathbf{a} \\
\beta_{1}, \beta_{2}, \mathbf{b}
\end{array} \mid x\right) \geq 0
$$

for all $x \in \mathbb{R}$. 
P r o o f. Indeed, according to [10, Theorem 1]

$$
{ }_{p} F_{p+1}\left(\begin{array}{c}
\alpha, \mathbf{a} \\
\beta_{1}, \beta_{2}, \mathbf{b}
\end{array} \mid x\right)=\frac{\Gamma(\mathbf{b})}{\Gamma(\mathbf{a})} \int_{0}^{1}{ }_{1} F_{2}\left(\begin{array}{c}
\alpha \\
\beta_{1}, \beta_{2}
\end{array} \mid x t\right) G_{p-1, p-1}^{p-1,0}\left(t \mid \begin{array}{c}
\mathbf{b} \\
\mathbf{a}
\end{array}\right) \frac{d t}{t}
$$

for $\mathbf{a}>0$ and $\psi=\sum_{k}\left(b_{k}-a_{k}\right)>0$, and

$$
\begin{aligned}
{ }_{p} F_{p+1}\left(\begin{array}{c}
\alpha, \mathbf{a} \\
\beta_{1}, \beta_{2}, \mathbf{b}
\end{array} \mid x\right)=\frac{\Gamma(\mathbf{b})}{\Gamma(\mathbf{a})} & {\left[{ }_{1} F_{2}\left(\begin{array}{c}
\alpha \\
\beta_{1}, \beta_{2}
\end{array} \mid x\right)\right.} \\
+ & \left.\int_{0}^{1}{ }_{1} F_{2}\left(\begin{array}{c}
\alpha \\
\beta_{1}, \beta_{2}
\end{array} \mid x t\right) G_{p-1, p-1}^{p-1,0}\left(t \mid \begin{array}{c}
\mathbf{b} \\
\mathbf{a}
\end{array}\right) \frac{d t}{t}\right]
\end{aligned}
$$

for $\mathbf{a}>0$ and $\psi=0$. Hence, the claim follows [5, Theorem 6.1] in view of nonnegativity of the $G$ function in the integrand valid according to [10, Theorem 2] or [11, Property 9].

\section{Acknowledgements}

The research of the first author has been supported by the Russian Science Foundation under the project 14-11-00022. The research of the second author has been supported by the Spanish Ministry of "Economía y Competitividad" under the project MTM2017-83490-P and by the Universidad Pública de Navarra.

\section{References}

[1] H. Alzer, On some inequalities for the gamma and psi functions. Mathematics of Computation. 66, No 217 (1997), 373-389.

[2] G.E. Andrews, R. Askey and R. Roy, Special functions. Cambridge University Press, Cambridge (1999).

[3] R. Beals, Advanced Mathematical Analysis. Springer Science and Business Media, New York (1973).

[4] R. Beals and R. Wong, Special Functions and Orthogonal Polynomials. Cambridge Studies in Advanced Mathematics (No. 153), Cambridge University Press, Cambridge (2016).

[5] Y.-K. Cho and H. Yun, Newton diagram of positivity for ${ }_{1} F_{2}$ generalized hypergeometric functions. Integral Transforms and Special Functions. 29, No 7 (2018), 527-542.

[6] L. Comtet, Advanced Combinatorics. The Art of Finite and Infinite Expansions, Revised and enlarged edition. D. Reidel Publishing Company, Dordrecht-Holland/Boston-U.S.A. (1974).

[7] O. Costin and H.M. Friedman, Foundational aspects of divergent integrals. J. Func. Anal. 267 (2014), 4732-4752. 
[8] R. Estrada and R.P. Kanwal, Regularization, Pseudofunction, and Hadamard Finite Part. Journal of Mathematical Analysis and Applications. 141 (1989), 195-207.

[9] S.I. Kalmykov and D.B. Karp, Log-concavity and Turán type inequalities for the generalized hypergeometric function. Analysis Mathematica. 43 No 4 (2017), 567-580.

[10] D. Karp, Representations and inequalities for generalized hypergeometric functions. Journal of Mathematical Sciences. 207, No 6 (2015), 885-897.

[11] D. Karp and J.L. López, Representations of hypergeometric functions for arbitrary values of the parameters and their use. Journal of Approximation Theory. 218 (2017), 42-70.

[12] D. Karp and J.L. López, On a particular class of Meijer's $G$ functions appearing in fractional calculus. to appear in International Journal of Applied Mathematics. (2018).

[13] D. Karp and E. Prilepkina, Hypergeometric functions as generalized Stieltjes transforms. Journal of Mathematical Analysis and Applications. 393 No 2 (2012), 348-359.

[14] D. Karp and E. Prilepkina, Completely monotonic gamma ratio and infinitely divisible $H$-function of Fox. Computational Methods and Function Theory. 16, No 1 (2016), 135-153.

[15] D.B. Karp and E.G. Prilepkina, Applications of the Stieltjes and Laplace transform representations of the hypergeometric functions. Integral Transforms and Special Functions. 28, No 10 (2017), 710-731.

[16] D. Karp and E. Prilepkina, Hypergeometric differential equation and new identities for the coefficients of Nørlund and Bühring. SIGMA. 12 (2016), 052, 23 pages.

[17] D. Karp and E. Prilepkina, D.B.Karp and E.G.Prilepkina, Extensions of Karlsson-Minton summation theorem and some consequences of the first Miller-Paris transformation. Integral Transforms and Special Functions. (2018). DOI:10.1080/10652469.2018.1526793

[18] D. Karp and S.M. Sitnik, Inequalities and monotonicity of ratios for generalized hypergeometric function. Journal of Approximation Theory. 161 (2009), 337-352.

[19] A.A. Kilbas, M. Saigo, H-transforms and applications. Analytical Methods and Special Functions 9. Chapman \& Hall/CRC, Boca Raton (2004).

[20] V.S. Kiryakova, Generalized Fractional Calculus and Applications. Pitman Research Notes in. Math. Series 301, Longman Group UK Ltd. (1994). 
[21] V.S. Kiryakova, All the special functions are fractional differintegrals of elementary functions. J. Phys. A: Math. Gen. 30 (1997), 5085-5103.

[22] V.S. Kiryakova, A brief story about the operators of the generalized fractional calculus. Fractional Calculus and Applied Analysis. 11 (2008), 203-220.

[23] V.S. Kiryakova, From the hyper-Bessel operators of Dimovski to the generalized fractional calculus. Fractional Calculus and Applied Analysis. 17, No 4 (2014), 977-1000.

[24] Y.L. Luke, The special functions and their approximations. Volume 1. Academic Press, San Diego (1969).

[25] N.E. Nørlund, Hypergeometric functions. Acta Mathematica. 94 (1955), 289-349.

[26] F.W.J. Olver, D.W. Lozier, R.F. Boisvert and C.W. Clark (Eds.) NIST Handbook of Mathematical Functions. Cambridge University Press, Cambridge (2010).

[27] A.P. Prudnikov, Yu.A. Brychkov and O.I. Marichev, Integrals and series, Volume 3: More Special Functions. Gordon and Breach Science Publishers (1990).

[28] A.M. Sedletskii, Analytic Fourier Transforms and Exponential Approximations. I. Journal of Mathematical Sciences. 129, No 6 (2005). Russian original: Sovremennaya mathematika. Fundamentalnyie napravleniya. 5 (2003), 3-152.

${ }^{1}$ Far Eastern Federal University, Vladivostok, Russia

and

Institute of Applied Mathematics FEBRAS, Vladivostok, Russia

e-mail:dimkrp@gmail.com

Received: July 30, 2018

2 Dpto. de Estadística, Informática y Matemáticas

Universidad Pública de Navarra and INAMAT, Navarra, Spain

e-mail: j.l.lopez@unavarra.es 